\title{
Zur Geschichte der Aniridie
}

\section{History of Aniridia}

„Für mich beginnt die Medizin nicht von heut und ich halte es für unmöglich, darin zu Hause zu sein, wenn man sie nicht genetisch auffasst."

Rudolf Virchow (1821-1902)

Rudolf Virchows Ansicht, welcher vom Autor uneingeschränkt zugestimmt wird, gilt gleichermaßen für die Augenheilkunde und jede einzelne Erkrankung, in besonderem Maße aber vielleicht für die Aniridie, deren Geschichte daher näher betrachtet werden soll. Will man sich über die oft nur mühsam und unsicher zu findende Erstbeschreibung einer Erkrankung des ophthalmologischen Fachgebiets orientieren, tut man gut daran, zunächst bei Julius Hirschberg (1843-1925) in seiner epochalen „Geschichte der Augenheilkunde“ nachzulesen. Hirschberg verweist bez. der Aniridie jedoch nur auf L.A. Kraus, der 1832 den Begriff „Irideremie“ (nach griechisch „Einsamkeit“ bzw. „Mangel“) vorschlug, ab 1844 aber die Bezeichnung „Aniridia“ („Irislosigkeit“) bevorzugt haben soll, und auf eine Arbeit von Gescheidt [1]. Anton Gescheidt aus dem Kreis um Friedrich August von Ammon (17991861) in Dresden ging 1834 tatsächlich ausführlicher auf die Irideremie ein [2].

G. Rindfleisch aus Heidelberg zitierte 1891 in einer seiner Arbeiten zur Aniridie [3] den Artikel von Johann Christian Jüngken (1793-1875) „Ueber den angeborenen Mangel der Iris, bei einem dreijährigen Knaben“ von 1820 [4]. Diese Arbeit Jüngkens, in der dieser von einer „merkwürdigen angeborenen Mydriasis“ sprach, war allerdings nur die Übersetzung einer Arbeit des Engländers Alex Morison, die 1819 auf Französisch publiziert worden war [5]. Bei Morisons „Sur un enfant dont les yeux n'avaient point d'iris“ dürfte es sich nach allem, was bekannt ist, um eine der ersten, wahrscheinlich sogar die erste Beschreibung der Aniridie handeln. Dementsprechend ist diese Irisanomalie noch nicht in der 3., posthumen Auflage des bekanntesten Lehrbuchs der Augenheilkunde aus dem 18. Jahrhundert von Charles
St. Yves (1667-1731) erwähnt [6]. Auch im allerersten Lehrbuch der Augenheilkunde „Das ist Augendienst“ von Georg Bartisch (1535-1607) aus dem Jahre 1583 finden sich keine Hinweise auf die Aniridie [7]. Zwar hat Bartisch einen Patienten mit sehr weiten, aniridieähnlichen Pupillen gezeichnet, wollte damit aber wahrscheinlich nur den „schwarzen Star“ illustrieren, also seinerzeit nicht diagnostizierbare, funktionsmindernde Erkrankungen des Augenhintergrunds, die keine Verfärbung der Pupille, wie etwa die Katarakt das tut, hervorriefen.

Im Jahre 1841 gab Friedrich August von Ammon seine „Klinische Darstellungen der Krankheiten und Bildungsfehler des menschlichen Auges“ heraus [8]. Das Buch besticht noch heute durch seine brillanten Abbildungen. Ammon widmete sich in diesem Werk der „Irideremie“ - diese anatomisch eigentlich korrektere Bezeichnung blieb noch bis in das 20. Jahrhundert hinein parallel zur „Aniridie“ gebräuchlich mit mehreren Abbildungen ( $\bullet$ Abb. 1) und einigen Seiten Text, in denen er u.a. schrieb: „Dieselbe (Anmerkung: Tafel) enthält eine Reihe von Abbildungen eines höchst wichtigen Bildungsfehlers des Auges, des partiellen und des totalen angeborenen Irismangels (Irideremia). Dieser angeborene Fehler, der meistens an beiden Augen zugleich, selten an einem allein sich zeigt, und gar nicht selten erblich vorkommt, ward in verschiedenen Abstufungen beobachtet, sodass entweder gar keine Spur der Iris vorhanden ist, oder höchstens ein schmaler, mehr oder weniger regelmäßiger, peripherischer Saum derselben erscheint. [...] Auch in diesen Fällen partieller Irideremie beobachtet man den grauschwarzen Grund der Augen, schwaches Sehvermögen, Photophobie, Andeutung von Blepharospasmus; dabei ist die Conjunctiva oculi nicht selten gerötet. Höchst wichtig ist das Vorkommen von Verdunkelungen des Linsensystemes“ [8]. Ammon kannte also bereits mehrere wesentliche klinische Symptome und Befunde mit ihrer Variabilität sowie die Heredität. Vermutlich war er der Erste, der -

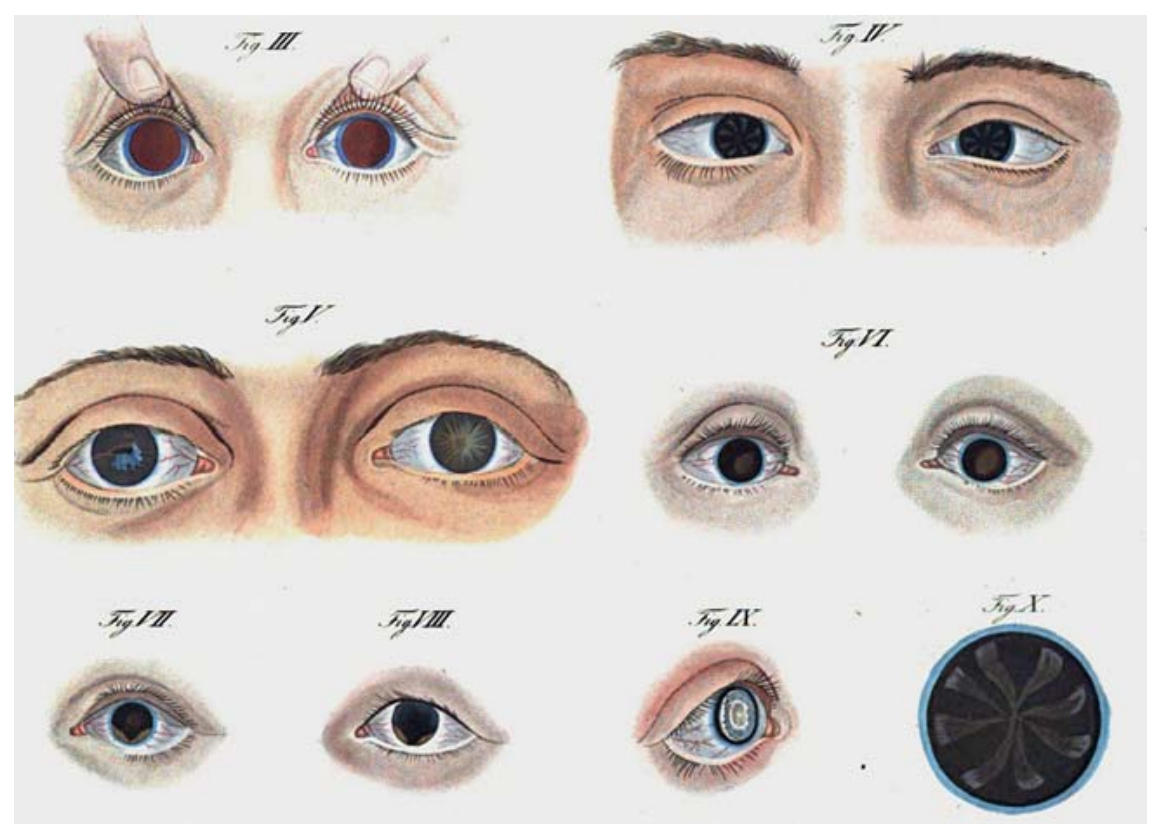

- Abb. 1 Mehrere zeichnerische Darstellungen von Aniridie unterschiedlichen Ausprägungsgrads, zumeist mit assoziierter Katarakt, 1841 [8]. 


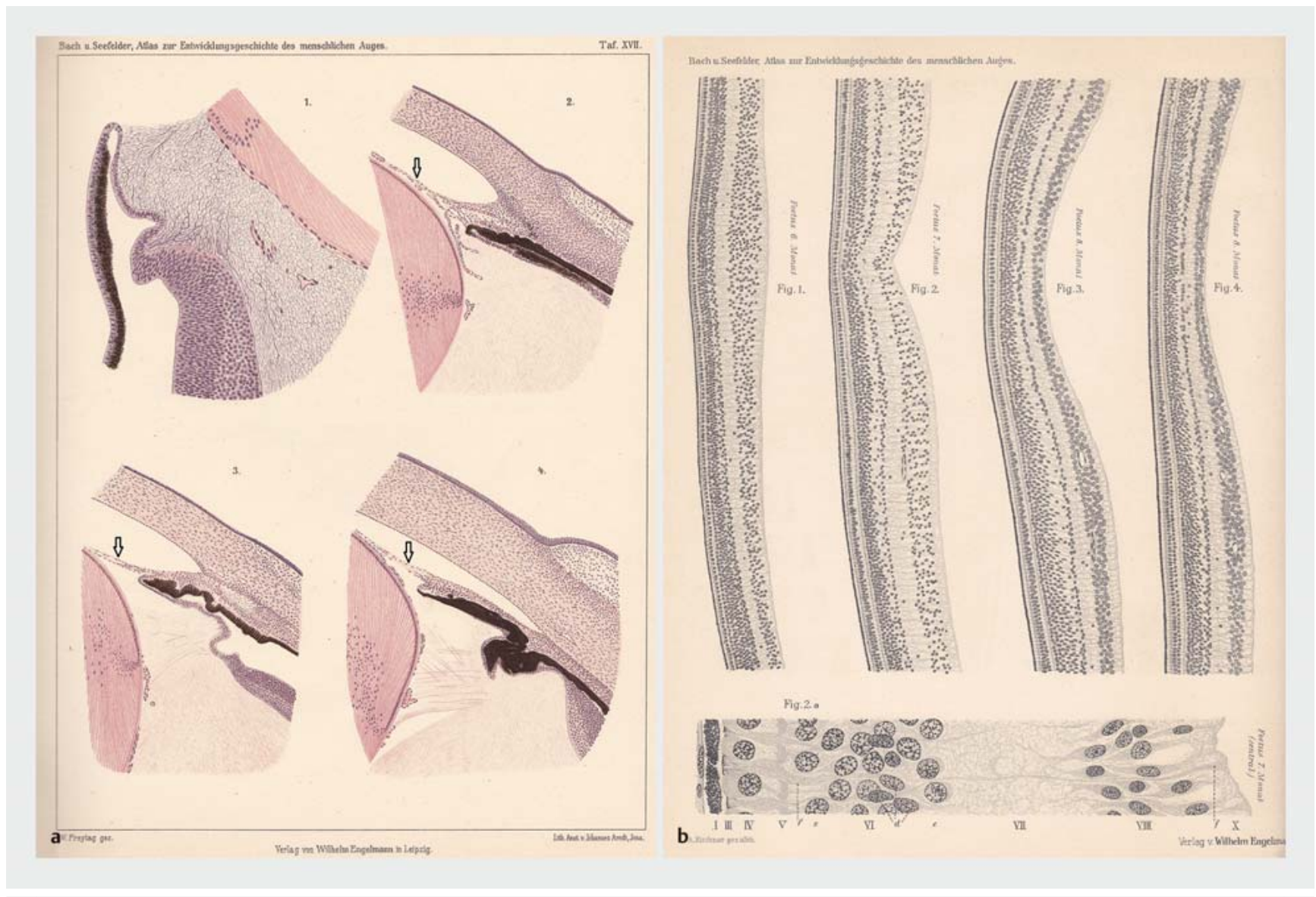

- Abb. 2 Abbildungen aus dem „Atlas der Entwicklungsgeschichte des menschlichen Auges“ [21]. a Entwicklung von Iris, Kammerwinkel, Ziliarkörper und Linse/Zonulasystem. Um 1900 wurde angenommen, dass die von der Iris zur Linse ziehende Pupillarmembran (Pfeil) die Ausbildung der Iris „mechanisch“ behindern und so zur Aniridie führen könnte. b Normale Entwicklung der Makula. Die foveale Depression beginnt sich im 6.7. Fetalmonat auszubilden. Seefelder schloss daraus, dass die Entwicklung der Makula beim Aniridiesyndrom vor dem 6. Monat der Schwangerschaft angehalten sein müsse.

1829 - eine Zergliederung und makroskopisch-pathologische Untersuchung eines Auges mit Aniridie durchführte [3]. 1858 befasste er sich noch einmal in einer separaten Monografie mit dieser Fehlbildung [9].

Ab der Mitte des 19. Jahrhunderts fand die Aniridie mehr und mehr Eingang in das ophthalmologische Schrifttum, und zwar sowohl in die Lehr- und Handbücher, die infolge der Verselbstständigung der Augenheilkunde (1850-1880) zunehmend auf den Markt kamen [10-13], als auch in die Fachzeitschriften, deren eigentlicher Beginn mit dem Erscheinen des „GraefeArchivs“ auf das Jahr 1854 zu datieren ist [3,14-16]. Dabei wurde nunmehr auch die Glaukomproblematik z.B. von Samelson und Hirschberg $[17,18]$ aufgegriffen. Hermann Pagenstecher (1844-1932) war wahrscheinlich der Erste, der 1871 mikroskopische Befunde von einem Auge mit Aniridie vorlegte $[3,19]$. Im Jahre 1900 gab es zur Aniridie also bereits ein recht umfangreiches Wissen, jedoch blieb die Pathogenese der Veränderungen zu dieser Zeit noch weitgehend unklar. Bis in das beginnende neue Jahrhundert hinein versuchte man, die Aniridie mit der ausbleibenden Entwicklung der radiären Irisgefäße und damit der Iris, der „mechanischen Unterdrückung“ der Irisentwicklung durch die Pupillarmembran/Tunica vasculosa lentis ( $\boldsymbol{A} \mathbf{A b} \mathbf{b} . \mathbf{2} \mathbf{a}$ ) oder durch eine intraokulare Entzündung während der Fetalzeit zu erklären [3,20]. Den entscheidenden Durchbruch zum Verständnis der Aniridie brachte Richard Seefelder ( Abb. 3), der sich seinerzeit als Oberarzt der Leipziger Klinik, ab 1919 als Direktor der Universitätsaugenklinik Innsbruck sehr intensiv mit der ophthalmologischen Embryologie und Teratologie befasste und 1914 gemeinsam mit dem Marburger Ordinarius Ludwig Bach (1865-1912) den „Atlas zur Entwicklungsgeschichte des menschlichen Auges“ veröffentlichte, ein Buch, das dank seiner wunderbaren zeichnerischen Abbildungen zumindest aus Sicht des morphologisch orientierten Ophthalmologen zu den schönsten Büchern der Augenheilkunde überhaupt gezählt werden kann ( $\triangleright$ Abb. 2) [21,22]. In sehr guter Kenntnis der normalen Embryologie des Auges setzte Seefelder mit „Die Aniridie als eine Entwicklungshemmung der Retina“ von 1909 einen entscheidenden Meilenstein in der Aufklärung dieser Erkrankung, obwohl er kasuistisch nur ein einziges Auge mit Aniridie, nämlich das einer an Tuberkulose verstorbenen 25-jährigen Frau, mikroskopisch untersucht 


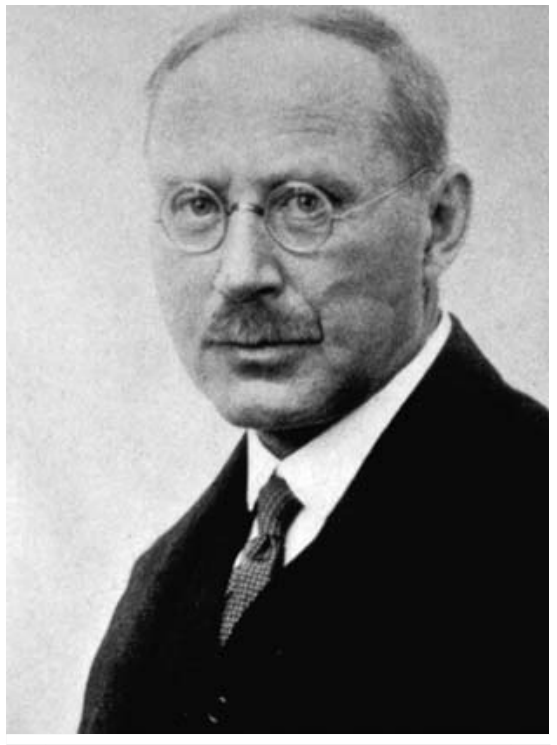

- Abb. 3 Richard Seefelder (1875-1949) (aus: Daxecker F. Richard Seefelder Vorstand der Univ.-Klinik für Augenheilkunde in Innsbruck von 1919 bis 1945. Klin Monatsbl Augenheilkd 2007; 224: 952-953).

hatte [20]. Die noch heute gültigen „Essentials" schilderte Seefelder in der klassischen Arbeit wie folgt: „Eine kurze Zusammenfassung der wichtigeren anatomischen Veränderungen ergibt das Vorhandensein eines schmalen Irisstumpfes in der ganzen Circumferenz des Bulbus, Verlegung eines großen Kammerwinkelabschnittes durch einen von der Iris zur Descemetschen Membran verlaufenden Fortsatz, Fehlen von Sphinkter und Dilatator in dem Irisrudiment, partielle Aplasie der Ciliarfortsätze, abnorme Kleinheit und kataraktöse Veränderungen der Linse, Degeneration der peripheren Netzhautzone, Verminderung der Zahl der Ganglienzellen in dem größten Netzhautabschnitte, dagegen temporal hinten Verdickung der Ganglienzellschicht weit über den makularen Bereich hinaus, Fehlen einer Fovea centralis, Verflüssigung des Glaskörpers.“

Seefelder hatte das Auge in Serie aufgeschnitten und so erstmals den histologischen Nachweis der Foveahypoplasie erbracht ( $\triangleright$ Abb. 4). Damit fand dann auch die seit Langem bekannte Visusminderung ihr wesentliches morphologisches Korrelat. Seefelders entscheidende Quintessenz lautete: „Der Name Aniridie oder Iridere-

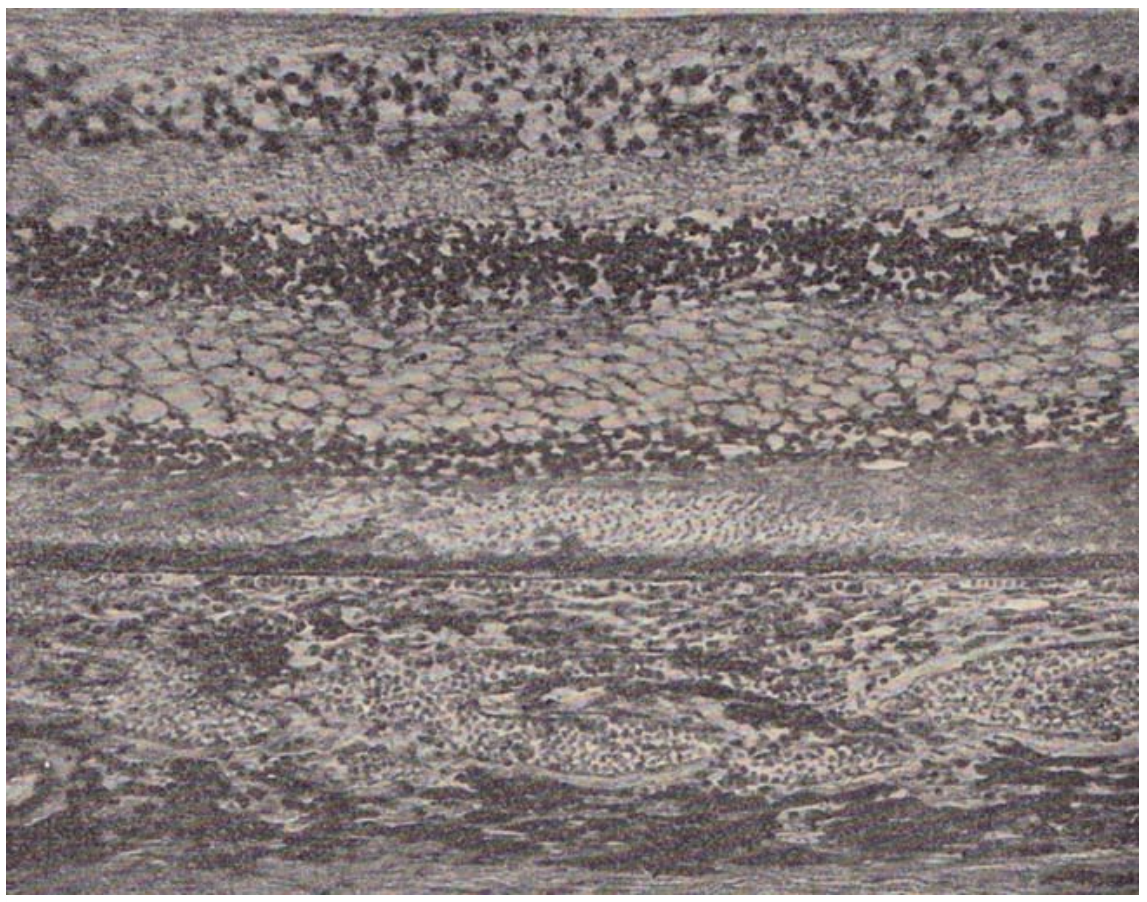

- Abb. 4 Histologie der Makulahypoplasie, d.h. der fehlenden fovealen Depression, aus der Originalarbeit Richard Seefelders [20].

mie bezeichnet nur die augenfälligste Erscheinung unter verschiedenen an Zahl und Intensität in den einzelnen Fällen wechselnden Anomalien ein und desselben Auges. Nach dieser Definition ist die Irideremie [...] nicht als ein selbstständiger Typus unter den Missbildungen des Auges, sondern nur als ein Symptom einer Entwicklungsstörung anzusehen, welche die ganze junge Augenanlage in mehr oder weniger empfindlicher und wahrnehmbarer Weise betroffen hat."

Da Seefelder von der normalen Embryologie her wusste, dass sich die Fovea erst ab dem 6 . Fetalmonat ausbildet ( $\bullet$ Abb. 2 b), schlussfolgerte er, dass es sich bei der Aniridie um eine Entwicklungsverzögerung bzw. -hemmung des inneren Blattes sowie des vorderen Teiles des äußeren Augenblatts des Augenbechers handeln müsse, welche vor dem 6. Fetalmonat wirksam wird. Mit Seefelder sollte man heute korrekterweise nicht von „Aniridie“ sondern von „Aniridiesyndrom“ [23], morphologisch präziser sogar von „Irideremiesyndrom" sprechen.

Während der Zeit des Nationalsozialismus wurde diskutiert, ob die Aniridie, insbe- sondere wenn sie mit Nystagmus, hochgradiger Visusminderung und Glaukom einherging, der „erblichen Blindheit“ zuzuordnen sei, die nach $\S 1$, Absatz 2 des „Gesetzes zur Verhütung erbkranken Nachwuchses“ vom 14. Juli 1933 einen Sterilisationsgrund darstellte [24]. Ob und wie viele Unfruchtbarmachungen bei Menschen mit Aniridie von 1933 bis 1945 tatsächlich erfolgten, ist unbekannt.

Nach Ida Mann (1893-1983), der großen britisch-australischen Ophthalmoembryologin und -teratologin [25], liegt der Beginn der Entwicklungshemmung beim Aniridiesyndrom im 3./4. Schwangerschaftsmonat [26]. In ihren „Developmental abnormalities of the eye" gab Mann zeichnerisch die Iris von zahlreichen Angehörigen einer Familie mit Aniridie wieder und verdeutlichte so, welche große Variabilität der Irismangel - vom nahezu kompletten Fehlen bis zu nur eher dezenten, kaum auffälligen Defekten des Irisstromas - haben kann [27]. Vermutlich sind in der Vergangenheit in Unkenntnis dieser Abortivformen zahlreiche Aniridiepatienten nicht als solche erkannt worden. Der Autor schließt sich diesbezüglich nicht aus. 


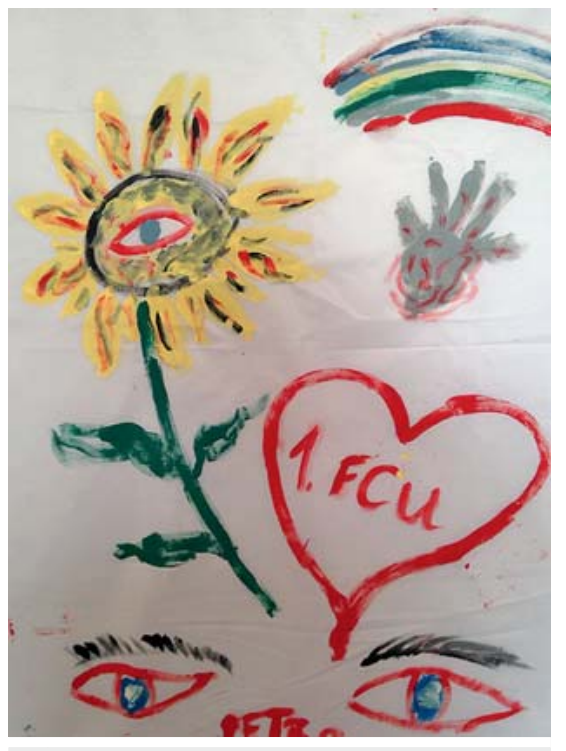

- Abb. 5 Bild aus der Aktion "gemeinsam Sehen - Union leben“, zu der u. a. auch Produktion und Verkauf einer Schallplatte gehörten. Kindergarten- und Schulkinder sowie Erwachsene aus Berlin und Brandenburg malten im September 2013 mehrere 100 Bilder zu den Themen „Union“ und „Auge“. Die Bilder wurden im Stadion „An der alten Försterei“ während des Heimspiels gegen Fortuna Düsseldorf an den Banden aufgehängt und anschließend verkauft. Die Aktion erlöste unmittelbar fast $19000 €$ und generierte mittelbar weitere Spenden zugunsten des Selbsthilfevereins. Die Gelder kamen der Aniridieforschung in Homburg zugute. Die Malerin „Petra“ ist dem Autor unbekannt.

Der genetisch sehr interessierte Alfred Vogt (1879-1943) lieferte im 3. Band seines Atlas der Spaltlampenmikroskopie von 1942 einen größeren, die autosomaldominante Vererbung demonstrierenden Stammbaum einer Familie mit Aniridie sowie klinische und - die Makulahypoplasie bestätigend - histologische Abbildungen [28]. Günter Badtke (1910-1967) behandelte die Aniridie in seinem großartigen Werk von 1961 auf 12 Seiten unter Zusammenfassung des seinerzeitigen Wissensstands [26]. Erst 3 Jahre später, 1964, wurde die Assoziation von Aniridie und Wilms-Tumor (Max Wilms, 1867-1918, ab 1910 bis zu seinem Tod Ordinarius für Chirurgie in Heidelberg) publiziert. Robert Miller und Mitarbeiter hatten bei 440 Kindern und Jugendlichen mit Nephroblastom festgestellt, dass die Frequenz der
Aniridie 1:73 betrug während sie in der Gesamtbevölkerung nur bei höchstens 1:47000 liegt. Auch wurden gehäuft Fehlbildungen des Urogenitalsystems beobachtet [29]. So wurde das WAGR-Syndrom als Kombination von Wilms-Tumor, Aniridie, genitalen Anomalien sowie geistiger Retardierung formuliert [29,30]. Fortan kam den Augenärzten die große Verantwortung zu, vor allem bei sporadischer Aniridie an den Wilms-Tumor zu denken und diesen kinderärztlicherseits ausschließen zu lassen [31, 32]. Der wesentliche genetische Defekt beim Aniridiesyndrom bzw. WAGR-Syndrom im PAX6-Gen auf Chromosom 11p ist seit nunmehr 25 Jahren bekannt [33-35]. PAX6 ist ein „Entwicklungsgen “ und spielt bei der normalen Embryonalentwicklung nicht nur des Auges eine überragende Rolle. Mutationen dieses Gens erklären damit sehr gut die von Richard Seefelder beschriebene okuläre Entwicklungshemmung. Über die zahlreichen PAX6-Mutationen hinaus können Defekte auch anderer Gene zum Aniridiesyndrom führen [23]. Je nach Genotyp bildet sich eine unterschiedliche Form der Aniridie mit unterschiedlichen Systemmanifestationen aus, weshalb genetische Untersuchung und Beratung Teil der Betreuung von Aniridiepatienten sein sollten $[23,33]$.

Vor einigen Jahren haben Netland und Mitarbeiter die Häufigkeit der okulären Veränderungen beim Aniridiesyndrom in einer Gruppe von 83 Patienten zusammengestellt. Dabei war der Nystagmus nach den obligaten Irisdefekten mit $83 \%$ führend, gefolgt von der Katarakt mit $71 \%$, dem „trockenem Auge“ mit 53\%, dem Glaukom mit 46\%, der Keratopathie mit $45 \%$ und der Foveahypoplasie mit (mindestens) $41 \%$ [36]. Immer deutlicher wird heute, dass die Aniridie nicht nur ein komplexes und sehr variables, okuläres Fehlbildungssyndrom darstellt, sondern mit ganz verschiedenen, z. T. erst in den letzten Jahren in den Blickpunkt geratenen systemischen Veränderungen einhergehen kann $[23,36]$. Als Folge der PAX6-Mutation finden sich gar nicht selten Veränderungen an der Epiphyse („3. Auge“) sowie im hypothalamisch-hypophysären und interhemispärischen System, was z.B. zu Schlafstörungen und Übergewichtigkeit, aber auch zu Hörstörungen führen kann
[23,37-39]. Auch scheinen im Rahmen des Aniridiesyndroms gehäuft Hautekzeme vorzukommen.

Die Betreuung von Menschen mit Aniridiesyndrom erfordert die Kooperation der verschiedenen ophthalmologischen Subdisziplinen, vor allem bei WAGR-Syndrom zusätzlich mit anderen medizinischen Fachdisziplinen. Damit ist das Aniridiesyndrom eine typische „Zentrumskrankheit“, weshalb in Homburg/Saar das erste Zentrum für diese Entität etabliert wurde. Nichtsdestotrotz muss das Krankheitsbild unbeschadet seiner Seltenheit jedem Ophthalmologen auch in „Katarakt- und IVOM-Zeit“ geläufig sein, was nach Auskunft von Betroffenen nicht immer der Fall zu sein scheint und für gewisse Ausbildungsdefizite spräche, befand doch Manfred Tost schon 1986 „Zu den häufigsten und jedem Augenarzt wohlbekannten, typischen Missbildungen des Auges gehört die Aniridie“ [40]. Die Aniridie nahm im „Axenfeld“, welcher von 1909 bis 1992 in 13 Auflagen mit weitgehend konstanter Konzeption erschien, einen bescheidenen, aber über mehr als 8 Jahrzehnte gleich bleibenden Raum ein [41], und heutige Lehrbücher für Studierende der Medizin berücksichtigen selbstverständlich, wenn auch nur kursorisch, klinisches Bild, Symptomatik und die mögliche Assoziation mit dem Wilms-Tumor $[42,43]$. Die Zahl der Differenzialdiagnosen ist insbesondere bei beidseitigen Irisdefekten gering. Das im Laufe des Lebens meist progrediente Aniridiesyndrom ist nicht heilbar, aber zumindest teilweise behandelbar [44].

Um das Los der Betroffenen durch Begegnungen und Forschung zu verbessern, wurde 2011 der Verein „Aniridie-WAGR e.V.“ gegründet (http://www.aniridiewagr.de). Trotz ihrer noch jungen Historie hat die Selbsthilfegruppe schon Beachtliches geleistet so z. B. durch (Mit-)Ausrichtung des 3. Europäischen Aniridiekongresses im August 2016 in Duisburg, an dem zahlreiche namhafte Aniridieforscher aus dem In- und Ausland teilnahmen. Unterstützt wird die Initiative durch 3 Fußballvereine, nämlich den VfR Aalen, den MSV Duisburg und Union Berlin ( Abb. 5). Geschah dies zunächst aus einer persönlichen Betroffenheit heraus, so hat sich das Engagement der Fußball- 
vereine verstetigt. Beide Seiten dürften davon profitieren. Die Selbsthilfegruppe kann ihre Anliegen auf etwas „unkonventionellem“, aber direktem Weg in die Gesellschaft transportieren, der VfR, der MSV und Union gewinnen an Prestige, da vorbildliche Übernahme gesellschaftlicher Mitverantwortung sichtbar wird. Möge diese Symbiose Modellcharakter haben.

\section{Anmerkung}

Nach einem Vortrag am 27.08.2016 anlässlich des 3. Europäischen Aniridiekongresses in Duisburg.

\section{Interessenkonflikt}

Nein.

Autor

J. M. Rohrbach

Department für Augenheilkunde, Forschungsbereich Geschichte der Augenheilkunde/Ophthalmopathologisches Labor, Eberhard-Karls-Universität Tübingen

\section{Literatur}

[1] Hirschberg J. Geschichte der Augenheilkunde. Deutschlands Augenärzte von 18001850. In: Graefe A, Saemisch T, Hrsg. Handbuch der gesamten Augenheilkunde. 2. Aufl. 14. Band, 2. Abteilung. Leipzig: Wilhelm Engelmann; 1911: 284

[2] Gescheidt A. Die Irideremie, das Iridoschisma und die Corectopie, die drei wesentlichsten Bildungsfehler der Iris. J Chir Augenheilkd (C. F. von Graefe) 1834; 22: 267-300

[3] Rindfleisch G. Zur pathologischen Anatomie und Genese des angeborenen Irismangels. Graefes Arch Ophthalmol 1891; 37: 221252

[4] Jüngken C. Ueber den angeborenen Mangel der Iris, bei einem dreijährigen Knaben. J Chir Augenheilkd (C.F. von Graefe) 1820; 1: 381-382

[5] Morison A. Observation sur un enfant dont les yeux n'avaient point d'iris. Nouveau Journal de Médecine, Chirurgie, Pharmacie 1819; 6: 105-107

[6] St. Yves C. Nouveau traité des maladies des yeux. 3. Aufl. Amsterdam, Leipzig: Arkstee \& Merkus; 1767
[7] Bartisch G. Das ist Augendienst. Dresden: Matthes Stöckel; 1583

[8] Ammon FA. Klinische Darstellungen der Krankheiten und Bildungsfehler des menschlichen Auges, der Augenlider und der Thränenwerkzeuge. Berlin: G. Reimer; 1841: 5057

[9] Ammon FA. Acyclia irideremia und Hemiphakia congenita. Zur Lehre von den angebornen Krankheiten des menschlichen Auges. Dresden: 1858

[10] Wharton-Jones T. Manual of the principles and practice of ophthalmic medicine and surgery. Section IV. Congenital defects of the iris and pupil. London: John Churchill; 1847: 318-319

[11] Manz W. Angeborener Irismangel/Sehstörungen bei Irideremie. In: Graefe A, Saemisch T, Hrsg. Handbuch der gesamten Augenheilkunde. 2. Band. Anatomie und Physiologie. 2. Teil. Leipzig: Wilhelm Engelmann; 1876: 87-91

[12] Vossius A. Grundriss der Augenheilkunde. Leipzig, Wien: Franz Deuticke; 1888: 210211

[13] Fuchs E. Lehrbuch der Augenheilkunde. 9. Aufl. Leipzig, Wien: Franz Deuticke; 1903: 393-395

[14] Rittmann. Angeborener gänzlicher Mangel der Regenbogenhaut an beiden Augen. Klin Monatsbl Augenheilkd 1865; 3: 158-159

[15] Vossius A. Congenitale Anomalien der Iris. Klin Monatsbl Augenheilkd 1883; 21: 233237

[16] Rindfleisch G. Ein Fall von angeborener Irideremie und Colobombildung der Iris am anderen Auge. Graefes Arch Ophthalmol 1892; 38: $183-198$

[17] Samelson A. Angeborene Aniridie mit Sehnerven-Excavation. Klin Monatsbl Augenheilkd 1877; 15: 188-192

[18] Hirschberg J. Angeborener Irismangel mit späterer Linsen-Verschiebung wie Trübung und Drucksteigerung. Centralbl Augenheilkd 1888; $12: 13-14$

[19] Pagenstecher H. Pathologisch-anatomische Mittheilungen. Klin Monatsbl Augenheilkd 1871; 9: 425-427

[20] Seefelder R. Die Aniridie als eine Entwicklungshemmung der Retina. Graefes Arch Ophthalmol 1909; 70: 65-87

[21] Bach L, Seefelder R. Atlas zur Entwicklungsgeschichte des menschlichen Auges. Leipzig, Berlin: Wilhelm Engelmann; 1914

[22] Rohrbach JM. Brauchen wir Bibliotheken heute noch? Ein Plädoyer für die Erhaltung ophthalmologischen Kulturguts. Klin Monatsbl Augenheilkd 2016; DOI: 10.1055/s-0042106903
[23] Käsmann-Kellner B, Seitz B. Aniridiesyndrom. Ophthalmologe 2014; 111: 11451156

[24] Rohrbach JM. Augenheilkunde im Nationalsozialismus. Stuttgart: Schattauer; 2007: 127-138

[25] Potter D. The Ophthalmological Society of New Zealand 39th scientific conference, 1985. The beginning of the chase: the embryological career of Ida Mann. Aust N Z J Ophthalmol 1986; 14: 97-107

[26] Badtke G. Die Missbildungen der Iris/Aniridie. In: Velhagen K, Hrsg. Der Augenarzt. Stuttgart: Thieme; 1961: 252-263

[27] Mann I. Developmental abnormalities of the eye. 2nd ed. London: British Medical Association; 1957: 230-235

[28] Vogt A. Lehrbuch und Atlas der Spaltlampenmikroskopie des lebenden Auges, dritter Teil. Stuttgart: Enke; 1942: 850-853

[29] Miller RW, Fraumeni JF jr., Manning MD. Association of Wilms's tumor with aniridia, hemihypertrophy and other congenital malformations. N Engl J Med 1964; 270: $922-$ 927

[30] DiGeorge AM, Harley RD. The association of aniridia, Wilms' tumor, and genital abnormalities. Arch Ophthalmol 1966; 75: $796-$ 798

[31] Flanagan JC, DiGeorge AM. Sporadic aniridia and Wilms's tumor. Am J Ophthalmol 1969; 67: 558-561

[32] Ruprecht KW, Naumann GOH. Aniridie und Wilms-Tumor. Berichte der DOG 1978; 75 : 588-590

[33] Neuhaus C, Betz C, Bergmann C et al. Genetik der kongenitalen Aniridie. Ophthalmologe 2014; 111: 1157-1163

[34] Ton CCT, Hirvonen H, Miwa $\mathrm{H}$ et al. Positional cloning and characterization of paired boxand homeobox-containing gene from the aniridia region. Cell 1991; 67: 1059-1074

[35] Cvekl A, Callaerts P. PAX6: 25th anniversary and more to learn. Exp Eye Res 2016; DOI: 10.1016/j.exer.2016.04.017

[36] Netland PA, Scott ML, Boyle JW et al. Ocular and systemic findings in a survey of aniridia patients. J AAPOS 2011; 15: 562-566

[37] Hanish AE, Butman JA, Thomas F et al. Pineal hypoplasia, reduced melatonin and sleep disturbance in patients with PAX6 haploinsufficiency. J Sleep Res 2016; 25: 16-22

[38] Abouzeid H, Youssef MA, ElShakankiri N et al. PAX6 aniridia and interhemispheric brain anomalies. Mol Vis 2009; 15: 2074-2083

[39] Han JC, Liu QR, Jones MP et al. Brain-derived neurotrophic factor and obesity in the WAGR syndrome. N Engl J Med 2008; 359: 918-927 
[40] Badtke G, Tost M. Mißbildungen der Iris/Aniridie. In: Velhagen K, Hrsg. Der Augenarzt. 2. Aufl. Band XI. Leipzig: VEB Thieme; 1986: $468-480$

[41] Rohrbach JM. Von der Evolution eines ophthalmologischen Lehrbuchs: „Der Axenfeld“ 1909-1992. Klin Monatsbl Augenheilkd 2013; 230: 832-842

[42] Lang GK. Augenheilkunde. Stuttgart: Thieme; 1998: 206-207

[43] Hahn GA. Kurzlehrbuch Augenheilkunde. Stuttgart: Thieme; 2012: 96
[44] Seitz B, Käsmann-Kellner B, Viestenz A. Stadiengerechte Therapie der kongenitalen Aniridie. Ophthalmologe 2014; 111: 11641171

Bibliografie

DOI https://doi.org/10.1055/s-0042-117836

Online-publiziert 14.12.2016 | Klin Monatsbl Augenheilkd 2018; 235: 324-329 @ Georg Thieme Verlag KG Stuttgart · New York | ISSN 0023-2165
Korrespondenzadresse

Prof. Dr. Jens Martin Rohrbach

Department für Augenheilkunde, Forschungsbereich Geschichte der Augenheilkunde/Ophthalmopathologisches Labor Eberhard-Karls-Universität Tübingen Schleichstr. 6-12 72076 Tübingen

Tel.: + 49/(0) 7071/2984761

Fax: + 49/(0) 7071/294762

Martin.Rohrbach@med.uni-tuebingen.de

(๑) $\diamond \Theta_{\text {verain } 1}$ 